\title{
THE HOURGLASS EFFECT AND THE MEASUREMENT OF THE TRANSVERSE SIZE OF COLLIDING BEAMS BY LUMINOSITY SCANS
}

\author{
M. Venturini* and W. Kozanecki ${ }^{\dagger}$ \\ Stanford Linear Accelerator Center, Stanford University, Stanford, CA 94309
}

\section{Abstract}

The formulae relating the luminosity to the transverse beam sizes as determined by luminosity scans, are derived with the hourglass effect properly taken into account.

\section{INTRODUCTION}

If the length of two colliding bunches is sufficiently small compared to the values $\beta_{x}^{*}$ and $\beta_{y}^{*}$ of the betatron functions at the interaction point (IP), the luminosity per bunch crossing is given by:

$$
\mathcal{L}^{*}=\frac{N_{1} N_{2}}{2 \pi \Sigma_{x}^{*} \Sigma_{y}^{*}}
$$

where $N_{1}$ and $N_{2}$ are the number of particles in the two colliding bunches, and $\Sigma_{x}^{*}, \Sigma_{y}^{*}$ are, respectively, the convoluted horizontal and vertical bunch size at the IP.

However, if the bunch lengths are comparable to or larger than $\beta_{x}^{*}$ and $\beta_{y}^{*}$, the luminosity is a more complicated function because of the variation of the transverse beam size along the length of each bunch. This is due to the growth of the betatron functions away from the IP. As a result, the actual luminosity is smaller than the nominal value (1): this is known in the literature as the 'hourglass effect'. A formula for the reduction factor between the actual and the nominal luminosity can be found in $[1,2]$.

Because the dependence of the luminosity on the sizes and relative positions of the colliding bunches is calculable, one can extract IP beam-size information from luminosity measurements carried out as a function of the relative transverse separation of the two beams at the collision point. The subject of this paper is the derivation of the correct relationship between the actually produced luminosity and the apparent transverse sizes extracted from luminosity scans, with the hourglass effect properly taken into account.

\section{LUMINOSITY FORMULA}

Consider two beam distributions in space and time $\rho_{1}(x, y, z, t)$ and $\rho_{2}(x, y, z, t)$. The general formula for the luminosity associated with the collision of the two beams is given by

$$
\mathcal{L}=\int d \boldsymbol{x} d t \rho_{1} \rho_{2}\left[\left(\boldsymbol{v}_{1}-\boldsymbol{v}_{2}\right)^{2}-\frac{\boldsymbol{v}_{1} \times \boldsymbol{v}_{2}}{c^{2}}\right]^{\frac{1}{2}},
$$

\footnotetext{
*venturin@slac.stanford.edu

${ }^{\dagger}$ Permanent address: DAPNIA-SPP, CEA-Saclay, F 91191 Gif-surYvette, France.
}

where $\boldsymbol{v}_{1}$ and $\boldsymbol{v}_{2}$ are the two beam velocities [3]. Now let us specialize to the case of ultra-relativistic beams moving along the $z$ axis, experiencing head-on collisions (i.e. the two velocities are parallel and $\left|v_{1}\right|=\left|v_{2}\right| \simeq c$ ), and having a rigid gaussian distribution in all three space dimensions with a transverse offset $(i=1,2)$ :

$$
\begin{aligned}
& \rho_{i}(x, y, z \pm c t)=\frac{N_{i}}{\sqrt{(2 \pi)^{3}} \sigma_{x i} \sigma_{y i} \sigma_{z i}} \\
& \exp \left[-\frac{\left(x-\bar{x}_{i}\right)^{2}}{2 \sigma_{x i}^{2}}-\frac{\left(y-\bar{y}_{i}\right)^{2}}{2 \sigma_{y i}^{2}}-\frac{(z \pm c t)^{2}}{2 \sigma_{z i}^{2}}\right]
\end{aligned}
$$

Assume that the coordinate frame is set in such a way that $z=0$ corresponds to the IP. The transverse rms sizes of the two beams close to the IP varies with $z$ according to $\sigma_{x i}^{2}=\sigma_{x i}^{* 2}\left(1+z^{2} / \beta_{x i}^{* 2}\right)$, and $\sigma_{y i}^{2}=\sigma_{y i}^{* 2}\left(1+z^{2} / \beta_{y i}^{* 2}\right)$, where $\beta_{x i}^{*}, \beta_{y i}^{*}$, and $\sigma_{x i}^{*}, \sigma_{x i}^{*}$ are, respectively, the betatron functions and the rms transverse sizes of the two beams at the IP.

Having introduced the definitions of the convoluted beam sizes $\Sigma_{x}(z)=\sqrt{\sigma_{x 1}^{2}(z)+\sigma_{x 2}^{2}(z)}, \quad \Sigma_{y}(z)=$ $\sqrt{\sigma_{y 1}^{2}(z)+\sigma_{y 2}^{2}(z)}$, and $\Sigma_{z}=\sqrt{\sigma_{z 1}^{2}+\sigma_{z 2}^{2}}$, we can carry out the integration in the transverse variables in (2) with $\rho_{i}$ defined by (3) and obtain

$$
\begin{aligned}
\mathcal{L} & =2 c \int d z d t \rho_{1} \rho_{2} \\
& =\frac{2 N_{1} N_{2}}{\sqrt{(2 \pi)^{3}} \Sigma_{z}} \int_{-\infty}^{\infty} d z \frac{\exp \left(-\frac{2 z^{2}}{\Sigma_{z}}-\frac{\bar{x}^{2}}{2 \Sigma_{x}}-\frac{\bar{y}^{2}}{2 \Sigma_{y}}\right)}{\Sigma_{x} \Sigma_{y}}
\end{aligned}
$$

In the above expression $\bar{x}=\bar{x}_{1}-\bar{x}_{2}$ and $\bar{y}=\bar{y}_{1}-\bar{y}_{2}$ indicate the relative displacement of the centroids of the two colliding bunches in the tranverse plane. After changing the integration variable to $u=\sqrt{2} z / \Sigma_{z}$ and having defined $u_{x}$ and $u_{y}$ as

$$
\begin{aligned}
\frac{1}{u_{x}^{2}} & =\frac{\Sigma_{z}^{2}}{2 \Sigma_{x}^{* 2}}\left(\frac{\sigma_{x 1}^{* 2}}{\beta_{x 1}^{* 2}}+\frac{\sigma_{x 2}^{* 2}}{\beta_{x 2}^{* 2}}\right), \\
\frac{1}{u_{y}^{2}} & =\frac{\Sigma_{z}^{2}}{2 \Sigma_{y}^{* 2}}\left(\frac{\sigma_{y 1}^{* 2}}{\beta_{y 1}^{* 2}}+\frac{\sigma_{y 2}^{* 2}}{\beta_{y 2}^{* 2}}\right),
\end{aligned}
$$

we can rewrite

$$
\begin{aligned}
& \mathcal{L}(\bar{x}, \bar{y})=\frac{N_{1} N_{2}}{2 \pi \Sigma_{x}^{*} \Sigma_{y}^{*}} \int_{-\infty}^{\infty} \frac{d u}{\sqrt{\pi}} \frac{u_{x} u_{y}}{\sqrt{u_{x}^{2}+u^{2}} \sqrt{u_{y}^{2}+u^{2}}} \times \\
& \exp \left(-u^{2}-\frac{1}{2} \frac{\bar{x}^{2}}{\Sigma_{x}^{* 2}} \frac{u_{x}^{2}}{u_{x}^{2}+u^{2}}-\frac{1}{2} \frac{\bar{y}^{2}}{\sum_{y}^{* 2}} \frac{u_{y}^{2}}{u_{y}^{2}+u^{2}}\right),
\end{aligned}
$$


where we have emphasized the dependence of luminosity on the relative transverse offsets $\mathcal{L}=\mathcal{L}(\bar{x}, \bar{y})$. Notice that the quantities $u_{x}$ and $u_{y}$ are a measure of the betatron functions at the IP in units of the bunch length. The expression $\mathcal{L}_{0}$ for the actual luminosity at $\bar{x}=\bar{y}=0$,

$$
\mathcal{L}_{0}=\frac{N_{1} N_{2}}{2 \pi \Sigma_{x}^{*} \Sigma_{y}^{*}} \int_{-\infty}^{\infty} \frac{d u}{\sqrt{\pi}} \frac{e^{-u^{2}}}{\sqrt{1+u^{2} / u_{x}^{2}} \sqrt{1+u^{2} / u_{y}^{2}}},
$$

is identical to that reported by Furman in $[1,2]$. Simple inspection of integral (6) shows that $\mathcal{L}_{0}=\mathcal{L}^{*}$ in the limit $u_{x}, u_{y} \rightarrow \infty$, as expected.

\section{BEAM SIZE FROM LUMINOSITY SCANS}

Beam size measurements using luminosity scans $[4,5]$ exploit the dependence of luminosity on the transverse distance between the centroids of the two colliding bunches. For fixed $\bar{y}$ (ideally $\bar{y}=0$ ) one can measure the luminosity as a function of the distance $\bar{x}$ separating the two bunches in the horizontal plane. The rms of the resulting gaussian is an estimate of the horizontal convoluted beam size. Similarly, one can keep $\bar{x}$ fixed and determine the vertical convoluted size by varying $\bar{y}$. We will refer to the bunch sizes measured in this way as the 'apparent' $\Sigma_{x}^{\text {app }}$ and $\Sigma_{y}^{\text {app }}$. As we shall see in a moment these quantities coincide with $\Sigma_{x}^{*}$ and $\Sigma_{y}^{*}$ only in the limit of vanishing bunch lengths. Formally we have:

$$
\begin{aligned}
\left(\Sigma_{x}^{\mathrm{app}}\right)^{2} & =\frac{\int_{-\infty}^{\infty} d \bar{x} \mathcal{L}(\bar{x}, 0) \bar{x}^{2}}{\int_{-\infty}^{\infty} d \bar{x} \mathcal{L}(\bar{x}, 0)}, \\
\left(\Sigma_{y}^{\mathrm{app}}\right)^{2} & =\frac{\int_{-\infty}^{\infty} d \bar{y} \mathcal{L}(0, \bar{y}) \bar{y}^{2}}{\int_{-\infty}^{\infty} d \bar{y} \mathcal{L}(0, \bar{y})} .
\end{aligned}
$$

In evaluating (7) and (8) one needs to carry out the following integrals:

$$
\begin{aligned}
& \int_{-\infty}^{\infty} \frac{e^{-u^{2}}}{\sqrt{1+u^{2} / a^{2}}} d u=|a| e^{a^{2} / 2} K_{0}\left(\frac{a^{2}}{2}\right), \\
& \int_{-\infty}^{\infty} \frac{u^{2} e^{-u^{2}}}{\sqrt{1+u^{2} / a^{2}}} d u= \\
& \frac{|a|^{3}}{2} e^{a^{2} / 2}\left[K_{1}\left(\frac{a^{2}}{2}\right)-K_{0}\left(\frac{a^{2}}{2}\right)\right],
\end{aligned}
$$

where $K_{0}$ and $K_{1}$ are the modified Bessel functions. We obtain

$$
\begin{aligned}
& \left(\Sigma_{x}^{\mathrm{app}}\right)^{2}=\Sigma_{x}^{* 2}\left(1+\frac{F\left(u_{y}\right)}{u_{x}^{2}}\right), \\
& \left(\Sigma_{y}^{\mathrm{app}}\right)^{2}=\Sigma_{y}^{* 2}\left(1+\frac{F\left(u_{x}\right)}{u_{y}^{2}}\right) .
\end{aligned}
$$

Here we have defined the auxiliary function

$$
F(u)=\frac{u^{2}}{2}\left(\frac{K_{1}\left(u^{2} / 2\right)}{K_{0}\left(u^{2} / 2\right)}-1\right) .
$$

The function $F(u)$, plotted in Fig. 1, increases monotonically from $F(0)=0$ to $F(\infty)=1 / 2$. As expected, $\Sigma_{x}^{\text {app }}$ and $\Sigma_{y}^{\text {app }}$ are always larger than and become identical to $\Sigma_{x}^{*}$ and $\Sigma_{y}^{*}$ in the limit $u_{x}, u_{y} \rightarrow \infty$.

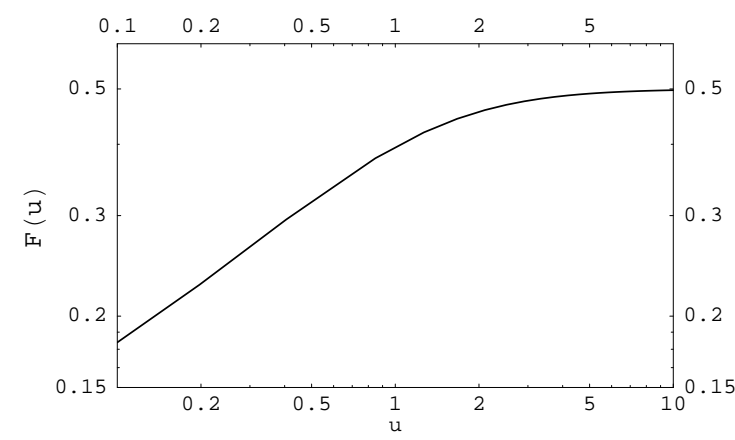

Figure 1: Function $F(u)$, defined in Eq. (13), vs. u.

\section{APPLICATION TO PEP-II}

The betatron functions at the IP and typical values for the longitudinal sizes for the electron (script ' - ') and positron (script '+') bunches in PEP-II are

$$
\begin{aligned}
& \beta_{x+}^{*}=\beta_{x-}^{*}=\beta_{x}^{*}=50 \mathrm{~cm}, \\
& \beta_{y+}^{*}=\beta_{y-}^{*}=\beta_{y}^{*}=1.25 \mathrm{~cm}, \\
& \sigma_{z+}=1.23 \mathrm{~cm} \\
& \sigma_{z-}=1.35 \mathrm{~cm} .
\end{aligned}
$$

From these numbers we obtain $\Sigma_{z}=\sqrt{\sigma_{z+}^{2}+\sigma_{z-}^{2}}=$ $1.82 \mathrm{~cm}$, and from Eqs. (4) and (5):

$$
\begin{aligned}
& u_{x}=\frac{\sqrt{2} \beta_{x}^{*}}{\Sigma_{z}} \simeq 38.8 \\
& u_{y}=\frac{\sqrt{2} \beta_{y}^{*}}{\Sigma_{z}} \simeq 0.968 .
\end{aligned}
$$

Because $u_{x}^{2} \gg 1$ one can evaluate the integral in (6) by taking $u^{2} / u_{x}^{2} \simeq 0$ in the denominator of the integrand and write [see Eq.(9)]:

$$
\mathcal{L}_{0} \simeq \frac{N_{1} N_{2}}{2 \pi \Sigma_{x}^{*} \Sigma_{y}^{*}} \frac{1}{\sqrt{\pi}} u_{y} e^{u_{y}^{2} / 2} K_{0}\left(u_{y}^{2} / 2\right) .
$$

The formula above gives the expression for the actual luminosity, i.e. the nominal (zero bunch length) luminosity degraded by the hourglass effect. The reduction factor by which we have to multiply the nominal luminosity $\mathcal{L}^{*}$ [see Eq. (1)] to obtain $\mathcal{L}_{0}$ is reported in Fig. 2 as a function of, $u_{y}$ (see also [1,2]). For PEP-II we have $\mathcal{L}_{0} / \mathcal{L}^{*} \simeq 0.85$ i.e. the actual luminosity is smaller than the nominal luminosity by about $15 \%$.

The actual luminosity $\mathcal{L}_{0}$ can also be expressed in terms of the 'apparent' convoluted beams sizes determined from 


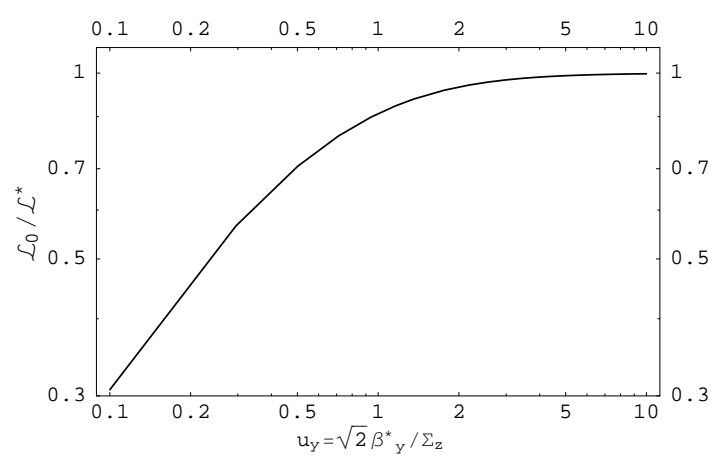

Figure 2: Plot of the hourglass reduction factor $\mathcal{L}_{0} / \mathcal{L}^{*}=$ $\pi^{-1 / 2} u_{y} e^{u_{y}^{2} / 2} K_{0}\left(u_{y}^{2} / 2\right)$ vs. $u_{y}$ [see Eq. (1) and (16)].

beam scans, by using Eqs. (11) and (12)

$$
\begin{aligned}
\Sigma_{x}^{* 2} & =\frac{\left(\Sigma_{x}^{\mathrm{app}}\right)^{2}}{1+F\left(u_{y}\right) / u_{x}^{2}} \simeq\left(\Sigma_{x}^{\mathrm{app}}\right)^{2} \\
\Sigma_{y}^{* 2} & =\frac{\left(\Sigma_{y}^{\mathrm{app}}\right)^{2}}{1+F\left(u_{x}\right) / u_{y}^{2}} \simeq \frac{\left(\Sigma_{y}^{\mathrm{app}}\right)^{2}}{1+1 /\left(2 u_{y}^{2}\right)} .
\end{aligned}
$$

In the expressions above we have made use of $F\left(u_{x}\right) \simeq$ $1 / 2$ and $F\left(u_{y}\right) / u_{x}^{2} \ll 1$, which hold because of (14) and (15). Therefore we can rewrite (16) in terms of the 'apparent' convoluted beam sizes as

$$
\mathcal{L}_{0}=\frac{N_{1} N_{2}}{2 \pi \Sigma_{x}^{\text {app }} \Sigma_{y}^{\text {app }}}\left[\frac{1}{\sqrt{\pi}} u_{y} e^{u_{y}^{2} / 2} K_{0}\left(u_{y}^{2} / 2\right)\right] \sqrt{1+\frac{1}{2 u_{y}^{2}}} .
$$

Often one uses the 'apparent' luminosity defined by

$$
\mathcal{L}^{\text {app }}=\frac{N_{1} N_{2}}{2 \pi \Sigma_{x}^{\text {app }} \Sigma_{y}^{\text {app }}}
$$

and computed from the measured $\Sigma_{x}^{\mathrm{app}}$ and $\Sigma_{y}^{\mathrm{app}}$, as an estimate of the actual luminosity. Eq. (19) shows that because of the hourglass effect this is only an approximation for $\mathcal{L}_{0}$. The term in the [ ] brackets in Eq. (19) is the reduction factor of Fig. 2 and is always smaller than one. On the other hand, the term with the square root in Eq. (19) is larger than one. It turns out that the latter always prevails so that the overall correction factor one has to apply to $\mathcal{L}^{\text {app }}$ in order to obtain the actual luminosity $\mathcal{L}_{0}$ is larger than one. We call this the 'hourglass augmentation factor'. It is plotted in Fig. 3 as a function of $u_{y}$.

For the PEP-II parameters reported at the beginning of this Section one obtains:

$$
\mathcal{L}_{0} / \mathcal{L}^{\mathrm{app}} \simeq 1.06
$$

\section{CONCLUSIONS}

In this paper we introduced three distinct quantities: the actual luminosity $\mathcal{L}_{0}$, Eq. (6), which is the luminosity produced when the bunches collide with no transverse offset; the nominal luminosity $\mathcal{L}^{*}$, Eq. (1), which depends only

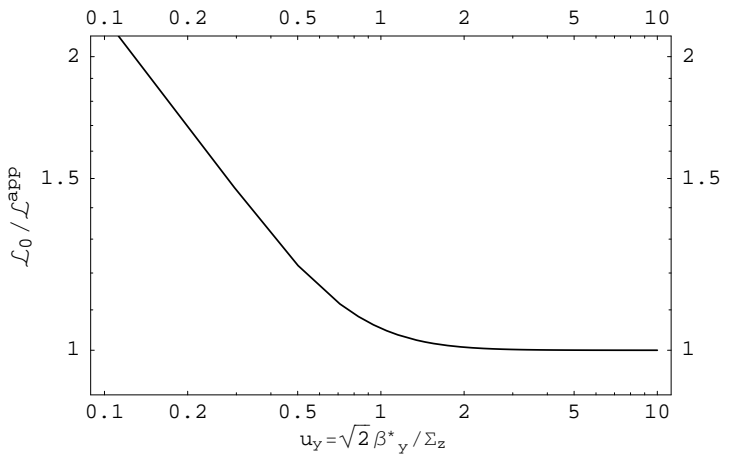

Figure 3: Hourglass augmentation factor $\mathcal{L}_{0} / \mathcal{L}^{\text {app }}=$ $\pi^{-1 / 2} u_{y} e^{u_{y}^{2} / 2} K_{0}\left(u_{y}^{2} / 2\right) \sqrt{1+1 /\left(2 u_{y}^{2}\right)} \quad$ vs. $\quad u_{y} \quad$ [see Eq. (19) and (20)].

on the nominal transverse beam sizes at the IP; and finally the apparent luminosity $\mathcal{L}^{\text {app }}$, Eq. (20), which is defined in terms of the 'apparent' beam sizes as measured by luminosity scans. If the hourglass effect is negligible these three quantities are all equal. However, if the betatron functions at the IP are smaller than, or of the same order as, the convoluted bunch length, the hourglass effect becomes important, and the actual luminosity $\mathcal{L}_{0}$ turns out to be smaller than the nominal $\mathcal{L}^{*}$, but larger than the apparent luminosity $\mathcal{L}^{\text {app }}$. The ratio $\mathcal{L}_{0} / \mathcal{L}^{*}$, called the hourglass reduction factor, is already known from the literature. The focus of this paper was to evaluate the ratio $\mathcal{L}_{0} / \mathcal{L}^{\text {app}}$, which was shown to be always larger than unity.

\section{ACKNOWLEDGMENTS}

Stimulating discussions with H. DeStaebler and J. Seeman provided the incentive for the work reported here. This work supported by DOE contract DE-AC03-76SF0051

\section{REFERENCES}

[1] M. Furman, The Hourglass Reduction Factor for Asymmetric Colliders, ABC-41, SLAC (1991).

[2] M. Furman, 'Luminosity', in A. Chao and M. Tigner, Handbook of Accelerator Physics an Engeneering, p. 247, World Scientific (1999).

[3] O. Napoly, Part. Acc. 40, 181-203 (1993).

[4] W. Kozanecki, et. al., Beam-Beam Experience at the PEP-II B-Factory, Proc. International Workshop on Performance Improvement of Electron-Positron Collider Particle Factories, Tsukuba (Japan), 21-24 September 1999.

[5] Y. Cai, Luminosity of Asymmetric $e^{+} e^{-}$Colliders with Coupling Lattices, SLAC-PUB-8479 (June 2000). 